\title{
The need for immunotherapy when neoadjuvant chemoradiotherapy achieves pathologic complete response in stage IIIB non-small cell lung cancer: a case report
}

\author{
Changsheng Lv, Lei Zhao, Yuntao Zhu, Peng Wang, Zhe Sun \\ Department of Thoracic Surgery, The First Affiliated Hospital of Dalian Medical University, Dalian, China \\ Correspondence to: Lei Zhao. The First Affiliated Hospital of Dalian Medical University, Dalian 116011, China. Email: pepper1999@163.com.
}

\begin{abstract}
The 5-year survival rate of patients with stage IIIB non-small cell lung cancer (NSCLC) range is $26 \%$. Pathological complete response (pCR) is the best outcome after treatment for stage IIIB NSCLC. For patients with stage IIIB NSCLC, concurrent chemoradiotherapy with a curative intent is currently the standard treatment. For patients who respond to treatment, this is followed by consolidation immunotherapy with durvalumab. However, because of the complex and diverse nature of stage IIIB NSCLC, standard treatment is not necessarily suitable for all patients; rather, individualized and precise treatment can maximize the benefits of patients. Herein, we report a case of a patient with stage IIIB lung squamous cell carcinoma (SCC) treated with neoadjuvant chemoradiotherapy after receiving all 6 cycles of treatment, the patient underwent video-assisted thoracoscopic surgery (VATS) right upper lobectomy, right middle partial lobectomy, right lower partial lobectomy, and systematic mediastinal lymph node dissection. Postoperative pathological section results showed a pCR. The patient did not continue to use immunotherapy as a consolidation treatment after surgery. He remained disease free until the latest follow-up a half year later. This case has led us to doubt whether immunotherapy with durvalumab is still needed for patients with pCR. However, more clinical trials are needed to provide stronger evidence.
\end{abstract}

Keywords: Chemoradiotherapy; non-small cell lung cancer (NSCLC); pathological complete response (pCR); case report

Submitted Feb 03, 2021. Accepted for publication Apr 21, 2021.

doi: 10.21037/apm-21-708

View this article at: http://dx.doi.org/10.21037/apm-21-708

\section{Introduction}

Lung cancer is a worldwide public health problem and the main cause of cancer-related mortality (1). The treatment of non-small cell lung cancer (NSCLC) depends on staging, which is based on the tumor $(\mathrm{T})$, node $(\mathrm{N})$, metastasis $(M)$ descriptors. Stage IIIB NSCLC indicates locally far advanced disease. Over the last few decades, the generally accepted standard therapy for patients with stage IIIB locally advanced NSCLC is combined modality chemoradiation. The international PACIFIC phase III clinical trial compared durvalumab and placebo in the treatment of stage III unresectable NSCLC with platinum doublet after chemoradiotherapy. The results showed that, compared with placebo, durvalumab's progression-free survival and overall survival (OS) was longer (2). Therefore, durvalumab consolidation therapy after chemoradiotherapy is currently considered as a standard therapy for stage IIIB NSCLC. Pathological complete response (pCR) is the optimal manifestation of neoadjuvant therapy efficacy. Melek et al. reported that the 5-year survival rate of pCR patients is about $70 \%$, and the recurrence rate is $20 \%$, which is lower than that of stage Ia NSCLC and similar to that of Ib NSCLC (3). Therefore, for patients who have achieved pCR, whether the continued use of durvalumab consolidation therapy can continue to provide benefit is still unknown. Herein, we reported a patient with initially inoperable IIIB squamous cell carcinoma (SCC) who achieved pCR to preoperative chemotherapy plus 

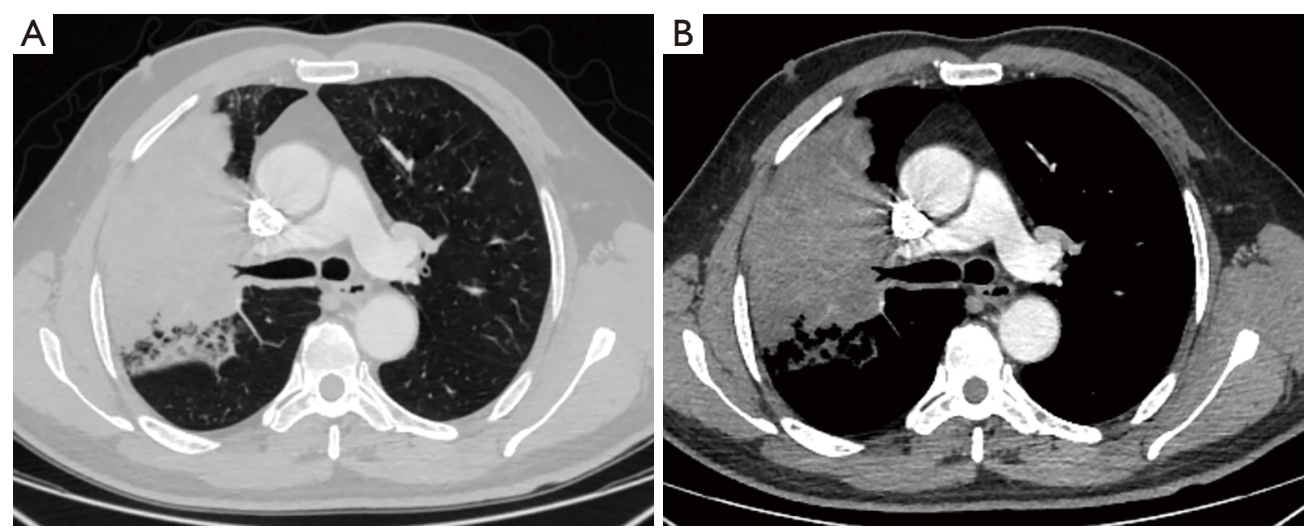

Figure 1 Chest computed tomography (CT) scan of the right hilar tumor before neoadjuvant therapy.

radiotherapy. Following this, he went on to achieve at least 6 months' disease-free survival (DFS) without durvalumab consolidation therapy or other treatments.

We present the following article in accordance with the CARE reporting checklist (available at http://dx.doi. org/10.21037/apm-21-708).

\section{Case presentation}

A 62-year-old Chinese male smoker (smoking index 800) presented to our hospital with a 2 months' history of a progressive cough and blood in the sputum for which antibiotics was ineffective. The patient underwent chest computed tomography (CT) scan, which showed a mass on the right hilum which had blocked the right upper lobe bronchus. The mass had also invaded part of the right middle lobe and part of the right lower lobe. The primary lesion was about $8.6 \mathrm{~cm}$ in diameter (Figure 1). Abdominal CT, brain magnetic resonance imaging (MRI), and bone scintigraphy found no evidence of metastasis in the abdomen, brain, or bone. Tumor marker analysis revealed the following: cytokeratin-19 fragment (CYFRA 21-1) $44.73 \mathrm{ng} / \mathrm{mL}$, carcinoembryonic antigen (CEA) $1.91 \mathrm{ng} / \mathrm{mL}$, squamous cell carcinoma antigen (SCCA) $0.97 \mathrm{ng} / \mathrm{mL}$, neuron specific enolase (NSE) $9.51 \mathrm{ng} / \mathrm{mL}$. Percutaneous lung puncture needle biopsy confirmed SCC. Immunohistochemical staining results revealed the following: cytokeratin 5/6 (CK5/6)+, cytokeratin 7 (CK7)-, novel aspartic proteinase A (Napsin A)-, $\mathrm{P} 40+$, and thyroid transcription factor-1 (TTF-1)-. According to the eighth edition lung cancer stage classification, staging of the patient was c-T4N2M0 (stage IIIB). There was no possibility of radical surgical resection. The patient's Eastern Cooperative
Oncology Group (ECOG) performance status was 0.

He had received chemotherapy [carboplatin (AUC6) plus paclitaxel $\left(175 \mathrm{mg} / \mathrm{m}^{2}\right)$, the cycle of chemotherapy is 21 days] as an initial treatment because concurrent chemoradiotherapy (cCRT) was not suitable for the wide extent and size of the tumor. After receiving 3 cycles of treatment, a CT scan of the chest showed that the size of the tumor was smaller than the size of the tumor before the initial treatment. We judged that cCRT could be performed at this time. Therefore, the tumor board decided to perform cCRT as the next treatment. After receiving all 3 cycles of cCRT (the chemotherapy regimen remained unchanged; the target dose of radiotherapy was $200 \mathrm{cGy}$ for 30 sessions, equaling a total dose of 6,000 cGy), chest CT scan showed that the tumor diameter and hilar lymph node size had further reduced (Figure 2). Positron emission tomography/ $\mathrm{CT}$ (PET/CT) showed a reduction in the volume of right upper lobe nodule and lymph node, but an increase in the metabolism of the lung nodule and right hilar lymph node. The situation was considered to be partial response (PR), according to the Response Evaluation Criteria in Solid Tumors (RECIST) version 1.1 (4). The toxicities reported during cCRT included thrombocytopenia (grade II) and nausea (grade II), according to the Common Terminology Criteria for Adverse Events (CTCAE) 5.0 criteria. Following multidisciplinary evaluation and discussion and 5 weeks after completing neoadjuvant cCRT, the patient underwent video-assisted thoracoscopic surgery (VATS) right upper lobectomy, right middle partial lobectomy, right lower partial lobectomy, and systematic mediastinal lymph node dissection. Pathological section samples revealed the size of the tumor bed was $3 \mathrm{~cm} \times 2 \mathrm{~cm} \times 1.5 \mathrm{~cm}$, and all the materials were collected to see the large areas of tumor 

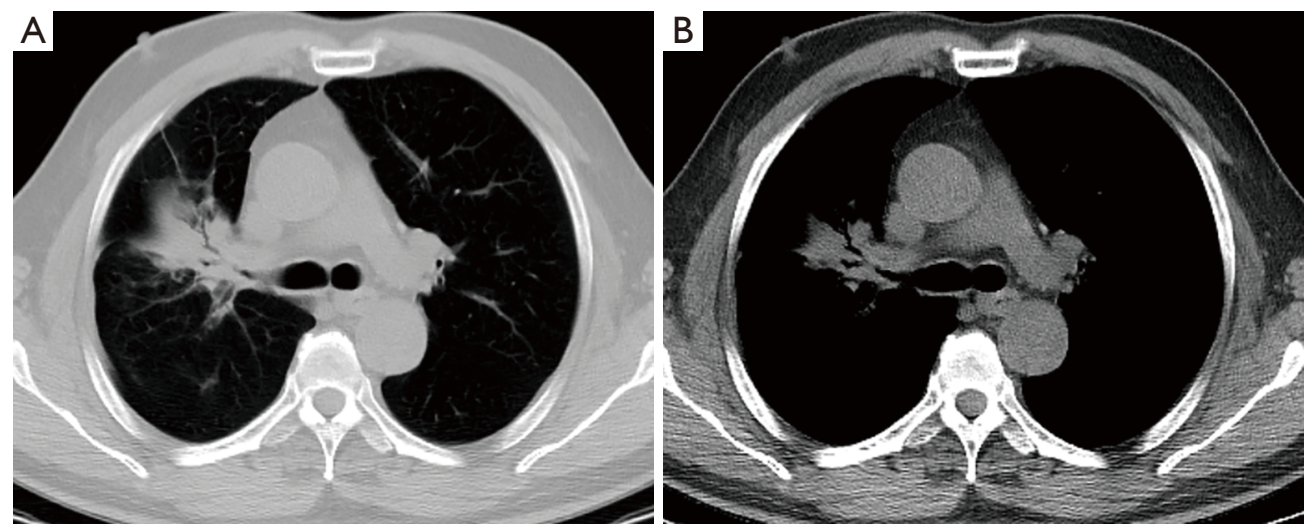

Figure 2 Chest computed tomography (CT) scan of the right hilar mass after neoadjuvant therapy.

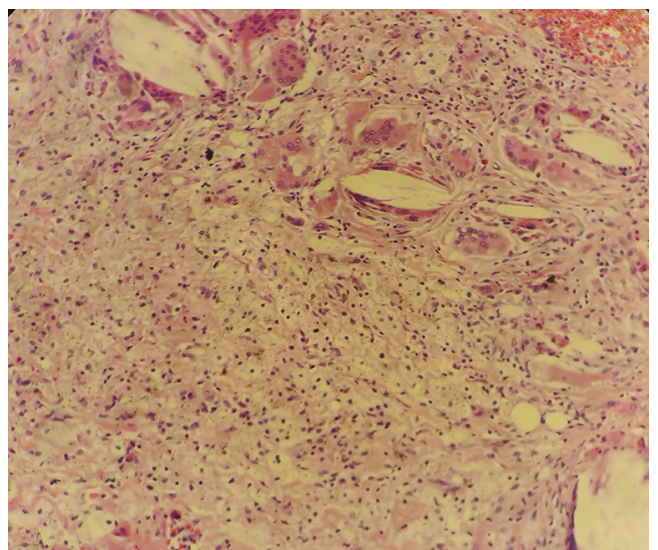

Figure 3 Postoperative pathological HE-stained picture with no surviving tumor cells (original magnification $\times 100$ ). HE, hematoxylin and eosin.

necrosis, cholesterol crystals and histiocytes, multinucleated cell aggregation, fibrous tissue proliferation, and lymphocyte infiltration; furthermore, no surviving tumor cells remained, which was consistent with complete response after treatment (Figure 3). Lymph nodes at levels 2, 3a, 4, 7, 8, and 12 were negative. The patient was discharged on postoperative day 6 after recovering with no perioperative complications, and there was no recurrence in the follow-up 6 months later.

All procedures performed in studies involving human participants were in accordance with the ethical standards of the institutional and/or national research committee(s) and with the Helsinki Declaration (as revised in 2013). Written informed consent was obtained from the patient for publication of this manuscript and any accompanying images.

\section{Discussion}

In recent years, immunotherapy represented by programmed cell death protein 1 (PD-1) or Programmed cell death protein 1 ligand (PD-L1) immune checkpoint inhibitors has revolutionized the treatment of many tumors. It kills tumor cells by modulating the body's own immune function and has a long-lasting effect. It can significantly improve the prognosis and has become the most potential anti-tumor treatment at present (5). In addition, immunotherapy and chemoradiotherapy have synergistic effects. Neoadjuvant chemoradiotherapy can cause changes in the local tumor microenvironment, increase the release and presentation of tumor antigens, and create an ideal platform for immune checkpoint inhibitors to function (6). It also brings more treatment options for patients with advanced NSCLC, constantly refreshing the survival time of patients with advanced NSCLC, and increasing the treatment benefits of NSCLC to New heights (7-9). The results of the phase III PACIFIC clinical trial established a new standard of treatment for patients with inoperable stage III NSCLC. The PACIFIC study was a double-blind phase clinical trial in patients with stage III unresectable NSCLC who received concurrent radiotherapy combined with platinum-containing chemotherapy ( $\geq 2$ cycles). Patients were randomized $2: 1$ to receive $10 \mathrm{mg} / \mathrm{kg}$ intravenous infusion of duvalizumab or placebo, twice a week, for up to 12 months. The follow-up data of the latest PACIFIC study was presented at the 2020 American Society of Clinical Oncology (ASCO) annual meeting. The results showed that the prospects for the application of durvalumab consolidation therapy after concurrent chemoradiotherapy are promising. The median OS time in the duvalizumab 
group reached 47.5 months, and the survival time was nearly 4 years. The placebo group's OS was only 29.1 months, for an extension of 18.4 months. The survival rate of the final 1.5 years improved [hazard ratio $(\mathrm{HR})=0.71$ ], and the risk of death decreased by $29 \%$. The 4 -year OS rate in the duvalizumab group was $49.6 \%$, which was a $13.6 \%$ improvement in survival compared to $36 \%$ in the placebo group, further suggesting that durvalumab may be an effective nonsurgical treatment in stage III patients.

Stage III NSCLC is a highly heterogeneous and highly complex tumor. Appropriate treatment for stage III has long been the subject of multidisciplinary controversy, and a fixed treatment model cannot give all patients the most survival benefit. According to current guidelines, patients with stage IIIB cannot be treated directly by surgical resection, while neoadjuvant therapy remains important part of the treatment in this population. Although pCR is not the primary goal of neoadjuvant therapy, it is one of the potential outcomes of neoadjuvant therapy (3). pCR refers to the absence of tumor cells in all specimens (ypTONOM0), which is an important prognostic factor for the treatment of stage IIIB NSCLC. In a large number of studies, pathological section samples from patients who underwent complete surgical resection and showed pCR after oncological treatment, and these patients' survival rates were equivalent to those of early-stage NSCLC patients. Furthermore, obtaining pCR is associated with lower local and remote recurrence rates, high progressionfree survival, and long-term survival (10-13). In reports of esophageal, rectal, breast, and bladder cancer, pCR has similar prognostic significance (14-18).

According to the eighth edition of the TNM classification, the current standard of treatment for patients with stage II and stage III pathological tumors is cisplatinbased dual chemotherapy after surgical resection. For locally advanced NSCLC patients with postoperative pathology yp-T0N0M0, despite the possibility of micrometastasis, there is no definitive evidence that postoperative adjuvant therapy is beneficial to such patients.

Our case report shows that a patient with stage IIIB lung SCC achieved pCR through neoadjuvant chemoradiation with no immunotherapy or other treatments in subsequent treatments. So far, he has achieved a DFS of at least 6 months. Although long-term follow-up is still needed to confirm that this treatment strategy is effective, this case still suggest that immunotherapy is perhaps not needed in the follow-up treatment of such patients.
In conclusion, the treatment of stage IIIB lung cancer has always been challenging. Fortunately, for stage IIIB lung cancer that initially responds to chemotherapy and radiation therapy, more patients have been cured through immunoconsolidation therapy. Unfortunately, the data cannot tell us how each person will respond to a given treatment method. Whether it is necessary to continue to immunotherapy in patients who have achieved pCR after chemoradiotherapy needs to be confirmed by more clinical trial evidence.

\section{Acknowledgments}

Funding: None.

\section{Footnote}

Reporting Checklist: The authors have completed the CARE reporting checklist. Available at http://dx.doi.org/10.21037/ apm-21-708

Conflicts of Interest: All authors have completed the ICMJE uniform disclosure form (available at http://dx.doi. org/10.21037/apm-21-708). The authors have no conflicts of interest to declare.

Ethical Statement: The authors are accountable for all aspects of the work in ensuring that questions related to the accuracy or integrity of any part of the work are appropriately investigated and resolved. All procedures performed in studies involving human participants were in accordance with the ethical standards of the institutional and/or national research committee(s) and with the Helsinki Declaration (as revised in 2013). Written informed consent was obtained from the patient for publication of this manuscript and any accompanying images.

Open Access Statement: This is an Open Access article distributed in accordance with the Creative Commons Attribution-NonCommercial-NoDerivs 4.0 International License (CC BY-NC-ND 4.0), which permits the noncommercial replication and distribution of the article with the strict proviso that no changes or edits are made and the original work is properly cited (including links to both the formal publication through the relevant DOI and the license). See: https://creativecommons.org/licenses/by-nc$\mathrm{nd} / 4.0 \%$. 


\section{References}

1. Siegel RL, Miller KD, Jemal A. Cancer statistics, 2019. CA Cancer J Clin 2019;69:7-34.

2. Antonia SJ, Villegas A, Daniel D, et al. Overall Survival with Durvalumab after Chemoradiotherapy in Stage III NSCLC. N Engl J Med 2018;379:2342-50.

3. Melek H, Çetinkaya G, Özer E, et al. Pathological complete response after neoadjuvant/induction treatment: where is its place in the lung cancer staging system?†. Eur J Cardiothorac Surg 2019;56:604-11.

4. Eisenhauer EA, Therasse P, Bogaerts J, et al. New response evaluation criteria in solid tumours: revised RECIST guideline (version 1.1). Eur J Cancer 2009;45:228-47.

5. Gettinger SN, Horn L, Gandhi L, et al. Overall Survival and Long-Term Safety of Nivolumab (Anti-Programmed Death 1 Antibody, BMS-936558, ONO-4538) in Patients With Previously Treated Advanced Non-Small-Cell Lung Cancer. J Clin Oncol 2015;33:2004-12.

6. Berman AT, Simone CB 2nd. Immunotherapy in locallyadvanced non-small cell lung cancer: releasing the brakes on consolidation? Transl Lung Cancer Res 2016;5:138-42.

7. Brahmer JR, Govindan R, Anders RA, et al. The Society for Immunotherapy of Cancer consensus statement on immunotherapy for the treatment of non-small cell lung cancer (NSCLC). J Immunother Cancer 2018;6:75.

8. Tsiara A, Liontos M, Kaparelou M, et al. Implementation of immunotherapy in the treatment of advanced non-small cell lung cancer (NSCLC). Ann Transl Med 2018;6:144.

9. Garon EB, Rizvi NA, Hui R, et al. Pembrolizumab for the Treatment of Non-Small-Cell Lung Cancer. N Engl J Med 2015;372;2018-28.

10. Pataer A, Kalhor N, Correa AM, et al. Histopathologic response criteria predict survival of patients with resected lung cancer after neoadjuvant chemotherapy. J Thorac Oncol 2012;7:825-32.

11. Mouillet G, Monnet E, Milleron B, et al. Pathologic complete response to preoperative chemotherapy predicts cure in early-stage non-small-cell lung cancer: combined

Cite this article as: Lv C, Zhao L, Zhu Y, Wang P, Sun Z. The need for immunotherapy when neoadjuvant chemoradiotherapy achieves pathologic complete response in stage IIIB nonsmall cell lung cancer: a case report. Ann Palliat Med 2021;10(4):4965-4969. doi: 10.21037/apm-21-708 analysis of two IFCT randomized trials. J Thorac Oncol 2012;7:841-9.

12. Betticher DC, Hsu Schmitz SF, Tötsch M, et al. Prognostic factors affecting long-term outcomes in patients with resected stage IIIA pN2 non-small-cell lung cancer: 5-year follow-up of a phase II study. Br J Cancer 2006;94:1099-106.

13. Lococo F, Cesario A, Margaritora S, et al. Long-term results in patients with pathological complete response after induction radiochemotherapy followed by surgery for locally advanced non-small-cell lung cancer. Eur J Cardiothorac Surg 2013;43:e71-e81.

14. Haisley KR, Hart KD, Nabavizadeh N, et al. Neoadjuvant chemoradiotherapy with concurrent cisplatin/5-

fluorouracil is associated with increased pathologic complete response and improved survival compared to carboplatin/paclitaxel in patients with locally advanced esophageal cancer. Dis Esophagus 2017;30:1-7.

15. Dinaux AM, Amri R, Bordeianou LG, et al. The Impact of Pathologic Complete Response in Patients with Neoadjuvantly Treated Locally Advanced Rectal Cancer-a Large Single-Center Experience. J Gastrointest Surg 2017;21:1153-8.

16. Prowell TM, Pazdur R. Pathological complete response and accelerated drug approval in early breast cancer. $\mathrm{N}$ Engl J Med 2012;366:2438-41.

17. Campbell JI, Yau C, Krass P, et al. Comparison of residual cancer burden, American Joint Committee on Cancer staging and pathologic complete response in breast cancer after neoadjuvant chemotherapy: results from the I-SPY 1 TRIAL (CALGB 150007/150012; ACRIN 6657). Breast Cancer Res Treat 2017;165:181-91.

18. Pokuri VK, Syed JR, Yang Z, et al. Predictors of Complete Pathologic Response (pT0) to Neoadjuvant Chemotherapy in Muscle-invasive Bladder Carcinoma. Clin Genitourin Cancer 2016;14:e59-e65.

(English Language Editor: J. Gray) 